\title{
THE USE OF STRUCTURE TRANSPLANTS WITH REVISION ENDOPROSTHETICS IN CONDITIONS OF SIGNIFICANT ACETABULAR OSTEOLYSIS
}

\author{
Karpukhin A.S. ${ }^{2}$, Tikhilov R.M. ${ }^{1}$, Tsybin A.V. ${ }^{1}$ \\ ${ }^{1}$ Russian Vreden institute of traumatology and orthopedics, Saint-Petersburg, e-mail: alex_tsybin@mail.ru; \\ ${ }^{2}$ Federal center of traumatology, orthopedics and endoprosthetics, Cheboksary, doc-karpukhin@yandex.ru
}

Aims.

To study long-term results of acetabular revisions with use of structural bone allografts in severe acetabular bone loss (2C, 3A and 3B by W. Paprosky classification).

\section{Materials and Methods}

One hundred eighty-three acetabular revisions with the use of bone allografts were performed; of these, structural bone allografts were used in 21 cases. In 7 cases, total revisions were performed; in 14 cases, isolated acetabular revisions were performed. In 20 cases $(95 \%)$, the reason for revision surgery was the aseptic failure of the acetabular or both components, in one case it was the sequel after deep infection. In three cases (14\%), before implantation of the acetabulum, bone grafting was performed with structural allografts - the femoral heads - with screw fixation. In 18 cases $(86 \%)$, structural allografts were tightly impacted in a previously prepared bone stock followed by the installation of the acetabular component.

Results.

This is an analysis of the long-term results of the use of structural allografts in 16 acetabular revisions with massive bone defects. The mean term follow up in the group was $75.35 \pm 31.1$ months (7-185 months). Of 16 cases of acetabular revision in the group, in three cases $(\mathbf{1 8 . 7 5 \% )}$ ) trabecular remodelling of allografts was noted, in three cases $(\mathbf{1 8 . 7 5 \%})$ there occurred trabecular incorporation, and in ten cases there were lack of changes in comparison with postoperative X-rays. In five cases $(\mathbf{3 1 . 2 5} \%)$, stage I resorption of transplants was recorded according to Sporer (lack of resorption); in six cases (37.5\%) stage II resorption (less than $25 \%$ ), in three cases $(\mathbf{1 8 . 7 5 \% )}$ stage III (25-50\%), and in two cases stage IV resorption was noted (over 50\% of transplant). Type I stability of Burch-Schneider cage (according to the Gill evaluation system) was revealed in three cases-lack of osteolysis around construction and screws. Mean change of the rotation centre was 0.23 mm; mean change of the inclination angle was $0.1 \mathrm{~mm}$. Type II stability was found in three cases-there was an increase of the osteolysis line upwards and medially. Mean change of the rotation centre was $9.6 \mathrm{~mm}$; mean change of the inclination angel was 10.9. Type III stability was not noted. Evaluation of the condition of press-fit fixation components (according to Moore) showed that in five cases there was lack of component migration, mean change of the rotation centre was $3.75 \mathrm{~mm}$, and mean change of the inclination angle was 1.4. In five cases, there was component migration, mean change of the rotation centre was $5.44 \mathrm{~mm}$, and mean change of the inclination angle was 14.06. In two cases out of 10, there was mechanical damage of the components (screw fractures)

Conclusions.

The use of structural allografts for filling of massive acetabular defects in acetabular revision is an effective method for the restoration of the bone stock, which allows to restore the centre of rotation and to install the acetabular component in its correct position.

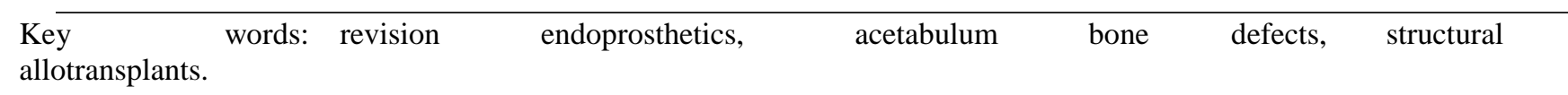

\section{INTRODUCTION.}

Total hip arthroplasty (THA) in patients with severe injuries and hip joint diseases is a high effective surgical procedure which promotes pain relief and improves function of the hip. According to the registers of arthroplasty (Swedish, Danish, Norwegian, and Finnish), around 40,000 primary arthroplasties are performed annually in Northern Europe and more than 1 million worldwide. In the next two decades, this figure is expected to increase by 2 times [1,2].

Due to a significant increase in the number of performed primary THA's, the number of revisions increase as well. The wear leads to the release of particles to the surrounding tissues, which can provoke tissue reactions, stimulate progressive osteolysis in the periprosthetic areas of 
the bone, lead to aseptic loosening of the components and massive bone defects. High-energy periacetabular fractures, surgical treatment of bone tumors, and periprosthetic infection can also lead to major acetabular bone defects [3,4].

Acetabular bone defects that occur during revision arthroplasty (removal of the acetabular component) can vary and reach a significant size. The deficit of bone stock can lead to serious technical difficulties for the implantation of the acetabulum in the condition of compromised columns, superior and medial walls of the acetabulum. Besides, as acetabular osteolysis progresses, the remaining bone stock cannot always provide correct installation of press-fit cups and screws (for primary mechanical stability of the component) and create favorable conditions for osteointegration, which correlates with the dates of the normal functioning of the endoprosthesis [5-10]. Presently, there are several surgical technics of acetabular revisions in patients with massive osteolysis for the restoration of acetabular bone stock.

A common peculiarity of the majority of methods is the use of allografts that differ by their structure (bone chips, structural grafts, including large osteochondral grafts).

The use of allogenous bone chips in revision arthroplasty has a comparatively rich history [6].

Due to its structure, this plastic material has good biological properties (transformation with the deformation of the bone basis). However, it lacks a supporting ability for implantation of the cups. This limits the range of indications for this method only to patients with cavitary bone defects and for the improvement of the congruence of the components of press-fit fixation. During the substitution of acetabular defects of the loaded sections of the acetabulum, it is obligatory to create a relative immobilization around the bone plate, which is achieved by the installation of revision systems, so-called "cages" (for example, Burch-Schneider cage, TMR), long-term unloading of the operated limb (from 2.5 months), and coordinated clinical-radiological observation of the patient's dynamics.

Structural bone allografts were widely used for the acetabular revision before the development and implementation of supporting blocks (tantalum constructions, Zimmer, Warsaw). Presently, they are widely used in patients with massive osteolysis for the implantation into the acetabular defects and the creation of support for the components press-fit fixation. However, the lack of long-term results, the unpredictability of tissue reactions, and the remaining deficit of bone mass (defect is substituted by the construction) indicate the possibility of the existence of alternative surgical methods for the restoration of the bone roof, in particular, implantation of structural allografts.

The use of biological material (allogenous bone structural grafts) is feasible with respect to future revisions. However, it has some drawbacks that include graft lysis. The dynamic of the changes in the structural graft is unpredictable in each individual case and correlates with the load on the sector of the acetabular component, wherein the area of the bone plate is located in the retro- 
acetabular space.

Jasty and Harris in the series of 38 observations with structural grafts in patients with massive acetabular osteolysis revealed a direct correlation between the degree of covering of the acetabular component with the structural graft, resorption of the later, and loosening of the acetabular component. The rate of unfavorable outcomes was $0 \%$ and, in 4 years, increased to $32 \%$ from the moment of revision [11].

Sporer et al. presented a similar study, wherein the group included 23 cases with the time of observation of 10 years [12]. The 10-year survival of acetabular components was $78 \%$ (the study endpoint was repeated revision for the acetabular aseptic loosening).

Lee et al. published the results of a long-term study that focused on the survival of the acetabular component after the revision in 74 patients with massive osteolysis using structural allografts (anterior column) [13]. The study results showed that survival of the acetabular component in 15 and 20 years after the surgery was $61 \%$ and $55 \%$, respectively (the study endpoint was repeated revision without specification of the reason).

These facts indicate that this method is questionable but, at the same time, has relatively high effectiveness for the acetabular revision with massive osteolysis, which provided the rationale for the present prospective study.

The study was aimed to evaluate long-term results of use of structural bone allografts in the conditions of the deficit of bone mass in the acetabulum during acetabular revision.

\section{MATERIALS AND METHODS.}

1. Clinical material.

One surgical team performed 183 acetabular revisions with use of bone allografts ( 21 of them were structural bone allografts) from May 25, 1999 to April 20, 2011. The group included 14 women and 7 men.

The study protocol followed guidelines for experimental investigation with human subjects in accordance with the Declaration of Helsinki and was approved by the ethics committee. Written informed consent was obtained from each patient (or an official representative) before the study.

The mean age of patients during the revision was $54.15 \pm 13.53$ years old (34-77).

In 7 cases, total replacement of endoprosthesis was performed and, in 14 cases, isolated acetabular revision.

In 20 cases (95\%), the revision was indicated because of aseptic loosening of the acetabular or both components, in one case, revision had to be performed because of deep surgical infection (the second stage of two-step treatment of deep surgical infection).

On average, before the present revision, the patients underwent $2.25 \pm 1.38$ operations (1$5)$.

The implanted constructions included 6 Duraloc components (DePuy), 6 Trilogy components (Zimmer), and 2 module tantalum trabecular components (TMT, Zimmer); the BurchSchneider cage (Zimmer) was installed in 7 cases (Figure 1).

In 3 cases (14\%), osteoplasty with a structural allograft (femoral head with screw fixation) 
was performed before the implantation of the acetabular component.

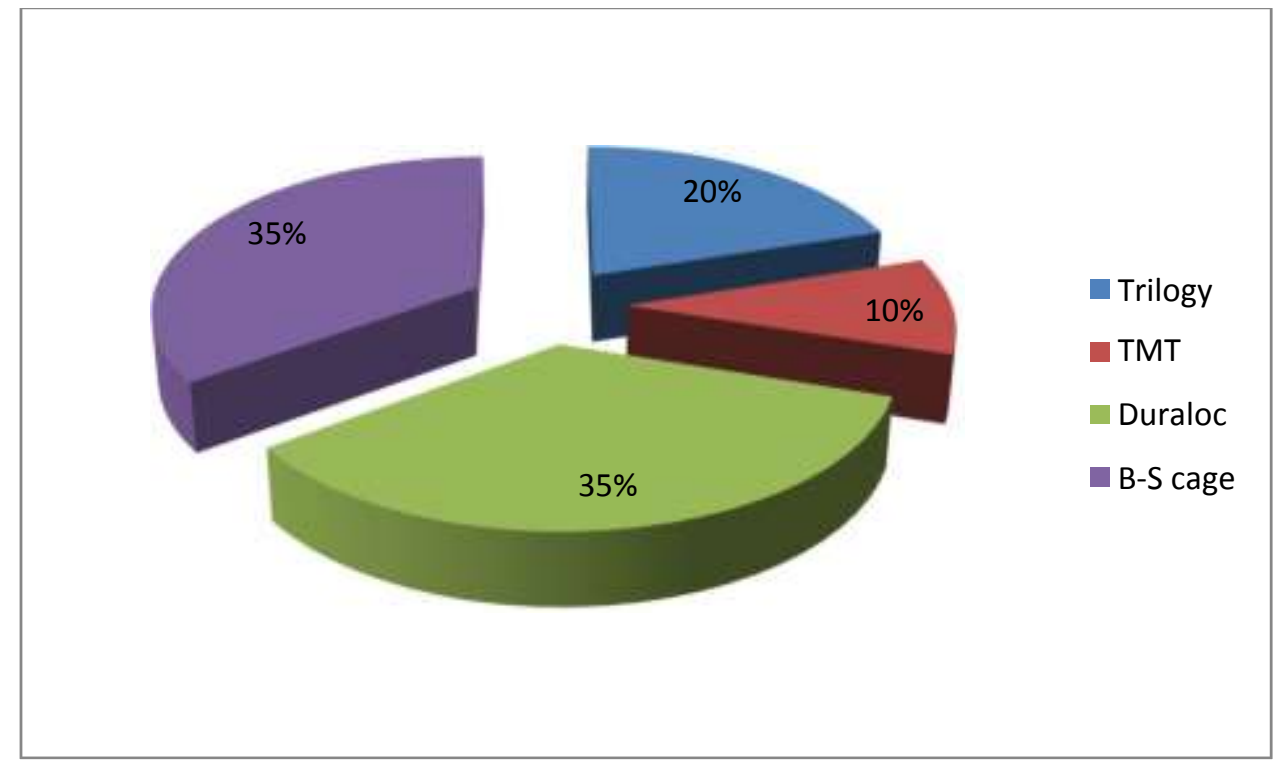

Figure 1. Acetabular systems implanted during acetabular component replacement.

In the rest 18 cases (86\%), the grafts were tightly impacted in the prepared implant bed with further installation of the endoprosthesis component. In all the cases, press-fit components were fixed with screws (an obligatory condition for the transformation of allograft bone) for reliable primary stability and unloading of the bone plate.

At admission, patients underwent a general clinical examination. The authors studied the anamnesis, archive records, and X-ray images. The joint puncture was performed with a further study of the punctate for the exclusion of deep surgical site infection.

The evaluation of the function of the hip joint was performed by the Harris hip score (before and after the operation) and the Oxford hip score [11].

2. Radiological evaluation.

At the admission, patients underwent a standard X-ray examination that included plain pelvic radiography, direct and axial projections of the hip joint. These studies allowed the authors to perform preliminary revision intervention and the evaluation of the size of components and the necessity for the osteoplastic replacement of acetabular defects for the recreation of the center of joint rotation.

The identification of the type of acetabular defect was performed by the Paprosky classification [14].

The evaluation of defects of the acetabulum in X-ray images was performed in the software Roman V 1.7.

After the calibration of the X-ray images, the authors made measurements by the 4 abovementioned criteria.

The distribution of patients by the type of the acetabular defect is presented in Table 1 . 


\begin{tabular}{|c|c|c|}
\hline $\begin{array}{c}\text { Type of } \\
\text { acetabular } \\
\text { defect }\end{array}$ & N & $\%$ \\
\hline $2 \mathrm{~A}-2 \mathrm{~B}$ & 3 & 14.3 \\
\hline $2 \mathrm{C}$ & 5 & 23.8 \\
\hline $3 \mathrm{~A}$ & 2 & 9.5 \\
\hline 3B & 11 & 52.4 \\
\hline
\end{tabular}

The obtained digital data was recorded in tables for further classification of the groups (by the criteria specified above) by the type of acetabular defect.

In the postoperative period, X-ray images were also analyzed in the software Roman V 1.7 by qualitative and quantitative parameters.

Quantitative evaluation of X-ray images included the measurement of the changes in the center of rotation and angle of inclination (horizontal deflection). The comparison of the obtained quantitative data in the dynamics and its comparison with the results of the qualitative analysis was performed (the latter will be presented further).

The migration of the acetabular component was evaluated in the software Roman V 1.7. The values were identified by the X-ray images and during the control examination ( 2 parameters were compared). The angle of inclination was identified in the same way.

The migration of the component was evaluated by the X-ray images (taken directly after the surgery and during the control examination) by comparing the distances between the lines that connect the tear-shaped figure and the perpendicular that goes through the center of the rotation of the operated joint.

The angle of inclination was calculated by the X-ray images with standard measurements (Figure 2). The sharp angle was identified between the line that connected teardrops and the line that connected endpoints (poles) of the acetabular component.

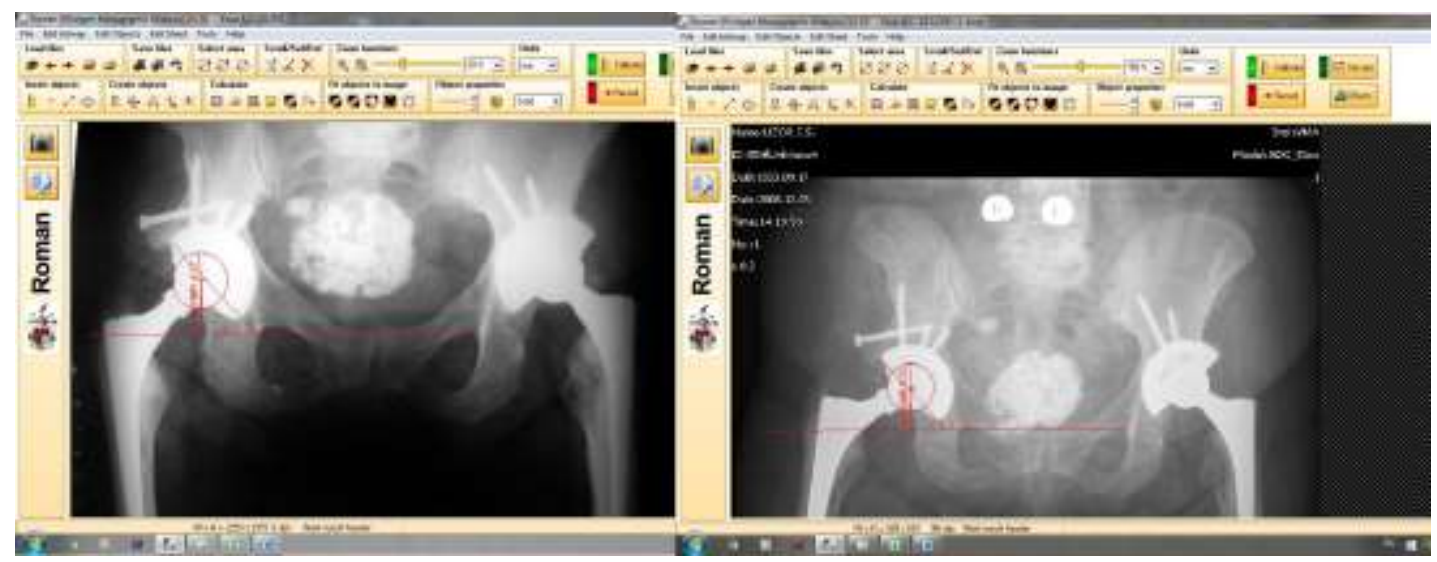

A

B

Figure 2. Examples of measurements: A. Directly after the surgery - the angle of inclination 47.3 
degrees, the angle of rotation $26.3 \mathrm{~mm}$; B. Control examination in 140 months - the angle of inclination 47.7 degrees, the center of rotation $24.8 \mathrm{~mm}$.

Additionally, the qualitative evaluation of the condition of the press-fit components by the Moose score was performed (see below).

Qualitative evaluation of X-ray images in the post-operative period was used for the estimation of the stability of the Burch-Schneider cage (Gill's criteria), press-fit components (Moor's criteria), and changes in the bones allografts (by Gross, by Sporer, by Gie).

\section{A. X-ray evaluation of the stability of the Burch-Schneider cage by Gill [15]:}

type I (possibly unstable) - the lines of osteolysis do not progress, no osteolysis around the screws;

type II (likely unstable) - the line of translucence increases upward and medially;

type III (definitely unstable) - cage fixing screws are broken, migration of the component is more than $5 \mathrm{~mm}$, which increased in the dynamics upwards, medially or around the screws.

The system of evaluation was based on 3 main criteria (osteolysis around the construction, osteolysis around the fixing screws and signs of mechanical damage of the screws. The obtained data (by each criterion) allowed the authors to evaluate the stability of the implanted cage.

\section{B. X-ray evaluation of the stability of press-fit fixation components. Five X-ray} characteristics by Moore [16]:

- superolateral support;

- inferomedial support;

- medial stress shielding;

- formation of radial trabecula;

- lack of X-ray lines of translucence.

The component was considered stable if there were 2 or more positive values of the specified characteristics.

The instability of the press-fit component was evaluated radiologically by the migration of the acetabular component for $4 \mathrm{~mm}$ and more, a change in the angle of inclination by 4 degrees and more, and the presence of 2 or more signs of osteointegration according to Moore's criteria and Gill's criteria.

\section{X-ray evaluation of the dynamics of the condition of bone allografts:}

1) Resorption by Gross [17]:

1. insignificant resorption (lysis $<1 / 3$ of the graft)

2. moderate resorption (lysis $1 / 3-1 / 2$ of the graft)

3. significant resorption (lysis $>1 / 2$ of the graft)

2) Resorption by Sporer [11]: 
$1.0 \%-$ no

2. $<25 \%$ mild degree

3. $25-50 \%$ moderate degree

4. $>50 \%$ significant degree

3) Transformation of the graft by Gie [18]:

1. no changes in comparison with post-operative X-ray images

2. trabecular incorporation

3. trabecular remodeling.

In the system of evaluation by Gie, trabecular incorporation differs from trabecular remodeling by the degree of "integration" of the trabeculae of bone tissue of the patients into the transplant structure. During the incorporation of the graft, the appearance of the trabeculae in the graft on the border between the bone and the graft is revealed on X-ray images. During the remodeling of the graft, it acquires the density similar to the patient's bone tissue, and the trabeculation spreads to all the massive of the structural donor material. The borderline in the area of the bone plate becomes diffuse (Figure 3).

The analysis of the X-ray images by the above-mentioned criteria (Gill, Moor, Sporer, and Gross) in small groups has a descriptive character and acquires statistical significance only in major samplings. However, the analysis of the published literature revealed the lack of similar studies in major groups of patients.

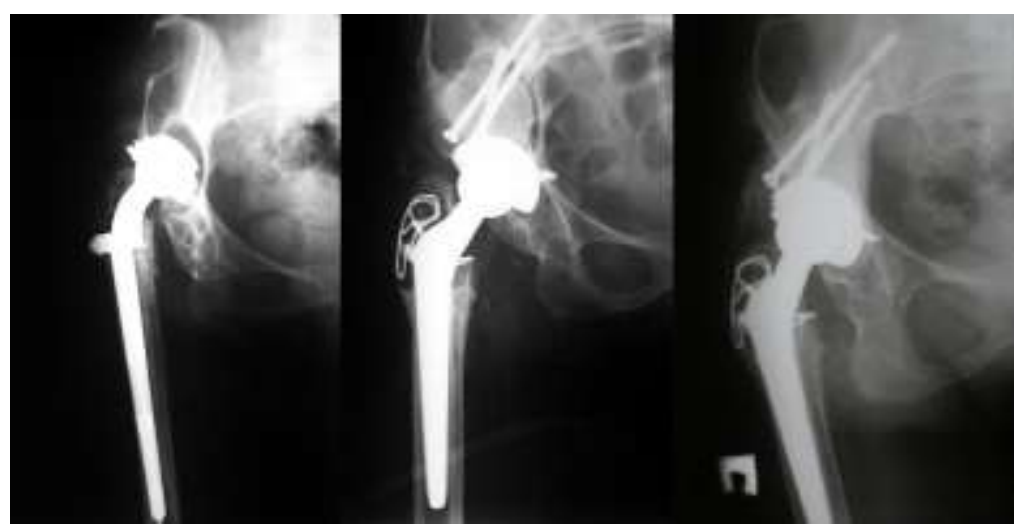

A

B

$\mathrm{C}$

Figure 3. X-ray image of Patient L., 50 years old: A. Before revision endoprosthetics. B. Right after the revision. C. Control X-ray imaging in 72 months. Trabecular remodeling of the graft by

Gie, 1 st degree of resorption by Gross (lysis < 1/3 of the graft), 2nd degree of resorption by Sporer (<25\% light degree).

RESULTS. The results were observed in 16 patients. The average period of observation in the group was $75.35 \pm 31.1$ months (7-185 months).

\section{Clinical evaluation of the results}

The average score by the Harris hip score was $24.5 \pm 6.3$ (18-34) before the surgery. During the control examination, it increased and reached $75 \pm 12.3$ points (12-91) by the Harris hip score and 
35.7 (17-48) points by the Oxford hip score.

The obtained data can be estimated as a good functional outcome.

\section{X-ray analysis}

\section{A. Evaluation of the changes in the grafts by Gie}

Out of 16 cases of revision prosthetics in the group, in 3 cases (18.75\%), trabecular remodeling of the grafts was observed; in 3 cases $(18.75 \%)$ - trabecular incorporation; and in 10 cases, the lack of changes was observed in comparison with postoperative X-ray images.

\section{B. Evaluation of the resorption of grafts by Sporer}

In 5 cases $(31.25 \%)$, 1st degree (no resorption) was observed, in 6 cases $(37.5 \%)-2$ nd degree of resorption (less than 25\%), in 3 cases (18.75\%) - 3rd degree (25-50\%), and in 2 cases 4th degree (more than $50 \%$ of the graft).

\section{Evaluation of the condition of the Burch-Schneider cage by Gill}

The 1st type of stability was revealed in 3 cases. There was no osteolysis around the construction and screws. The mean change in the center of rotation was $0.23 \mathrm{~mm}$, the mean change in the angle of inclination was $0.1 \mathrm{~mm}$.

The 2 nd type of stability was identified in 3 cases. There was an increase in the line of osteolysis upwards and medially. The mean change in the center of rotation was $9.6 \mathrm{~mm}$, the mean change in the angle of inclination was $10.9 \mathrm{~mm}$.

The 3rd type of stability was not registered.

\section{Г. Evaluation of the condition of press-fit components by Moore}

In 5 cases, there was a lack of migration of components. The mean change in the center of rotation was $3.75 \mathrm{~mm}$, the mean angle of inclination was $1.4 \mathrm{~mm}$.

In 5 cases, there was a migration of the components, the mean change in the center of rotation was $5.44 \mathrm{~mm}$, the mean angle of inclination was $14.06 \mathrm{~mm}$.

In 2 cases out of 10, there was a mechanical damage of the constructions (screws fractures).

\section{Complications}

In 5 cases, the observation in the dynamics revealed different complications. In 2 cases, deep surgical site infection was revealed that required two-step treatment. It was the use of structural grafts that provided an increase in the bone mass of the acetabulum.

In 3 cases, there was aseptic instability of the acetabular component that required repeated acetabular revision. One patient from the group died 9 years after the surgery because of comorbid pathology.

Thus, in 5 cases out of 16 (31.25\%), further revision interventions were required.

The presented cases of acetabular revision were significantly complicated by massive acetabular osteolysis. In $52.4 \%$ of cases, there were 3B defects by the Paprosky classification. The application of structural grafts provided successful restoration of the acetabular roof in $68.75 \%$ 
cases and allowed obtaining good mid-term results. In $31.25 \%$ of cases, the observed complications required repeated revision interventions. However, the chosen type of grafts provided the formation of the bone basis for "future" revisions, which, in the future, allowed the surgeons to use standard components of the endoprosthesis. Thus, it can be highlighted that the use of structural allografts in the conditions of massive osteolysis of the acetabulum can provide a feasible alternative for modern revision complexes and good mid-term results [19-21]. However, it requires further observation and follow-up of the long-term results.

\section{CONCLUSIONS.}

The application of structural allografts for the replacement of massive acetabular defects (2C, 3A, 3B by Paprosky) is an effective method of restoration of the acetabulum, provides the restoration of the center of rotation, and allows for the implantation of the acetabular component of the endoprosthesis in the proper position.

Use of structural allografts for filling massive acetabular defects during acetabular revision showed good mid-term results in $67.75 \%$ of cases.

Structural allografts for acetabular revisions are recommended for young patients because it allows surgeons to form the basis for "future" revision interventions.

\section{FINANCIAL SUPPORT AND SPONSORSHIP}

Nil.

\section{CONFLICTS OF INTEREST}

The authors declare no conflict of interest

SUPPLEMENTARY DATA (DOI)

\section{REFERENCES}

1. Pivec R., Johnson A.J., Mears S.C., et al. Hip arthroplasty. Lancet, 2012; vol. 380, pp. 1768- 1777, doi.org/10.1016/s0140-6736(12)60607-2.

2. Nguen Nam Min. Research and development of tools of ESCS of biomechanical objects: autoreferate of thesis of Candidate of Technical Sciences [Issledovanie i razrabotka instrumental'nykh sredstv SAPR biomekhanicheskikh ob"ektov: avtoreferat dissertatsii kandidata tekhnicheskikh nauk], St. Petersburg, 2015, 3 p.

3. Howie D.W., Neale S.D., Martin W. et al. Progression of periacetabular osteolytic lesions. The Journal of bone and joint surgery. American volume, 2012, vol. 94, no 16, pp. e1171-1176, doi 10.2106/jbjs.k.00877.

4. Karam J.A., Tokarski A.T., Ciccotti M. et al. Revision total hip arthroplasty in younger patients: 
indications, reasons for failure, and survivorship. The Physician and Sportsmedicine, 2012, vol. 40 no 4, pp. 96- 101, doi: 10.3810/psm.2012.11.1992.

5. Rudelli S., Honda E., Viriato S.P. et al. Acetabular Revision With Bone Graft and Cementless Cup. The Journal of Arthroplasty, 2009, vol. 24, no. 3, pp. 432-443, doi 10.1016/j.arth.2007.11.022.

6. Campana M., Milano G., Pagano E. et al. Bone substitutes in orthopaedic surgery: from basic science to clinical practice. Journal of Materials Science: Materials in Medicine. 2014; vol. 25, no 10, pp. 2445-2461, doi 10.1007/s10856-014-5240-2.

7. Shon W.Y., Santhanam S.S. Choi J.W. Acetabular Reconstruction in Total Hip // Arthroplasty Hip Pelvis, 2016, vol. 28, no 1, pp. 1-14. doi: 10.5371/hp.2016.28.1.1.

8. Regis D., Sandri A., Bonetti I. Acetabular Reconstruction with the Burch-Schneider Antiprotrusio Cage and Bulk Allografts: Minimum 10-Year Follow-Up Results. BioMed Research International, 2014, vol. 2014, pp. 1-9, doi: 10.1155/2014/194076.

9. Malhotra R., Kumar Y. Acetabular revision using a total acetabular allograft. Indian Journal of Orthopaedics, 2009, vol. 43, no 2, pp. 218-221, doi: 10.4103/0019-5413.50860.

10. Kmieć K., Dorman T., Andrzej G. et al. Early results of revision acetabular cup using antiprotrusio reconstruction rings and allografts. Indian Journal of Orthopaedics, 2015, vol. 49, no 3, pp. 317-322, doi: 10.4103/0019-5413.156205.

11. Jasty M., Harris W.H. Salvage total hip reconstruction in patients with major acetabular bone deficiency using structural femoral head allografts. The Journal of Bone and Joint Surgery. British volume, 1990; vol. 72-B, no 1, pp. 63-67, doi 10.1302/0301-620x.72b1.2298796.

12. Sporer S.M., O'Rourke M., Chong P. et al. The use of structural distal femoral allografts for acetabular reconstruction. Average ten-year follow-up. The Journal of Bone and Joint SurgeryAmerican Volume, 2005, vol. 87, no 4, pp. 760-765, doi 10.2106/00004623-200504000-00010.

13. Lee P.T., Raz G., Safir O.A. et al. Longterm results for minor column allografts in revision hip arthroplasty. Clinical Orthopaedics and Related Research, 2010, vol. 468, no 12, pp. 3295-3303, doi 10.1007/s11999-010-1591-2.

14. Paprosky W.G, Perona P.G, Lawrence J.M. Acetabular defect classification and surgical reconstruction in revision arthroplasty. A 6-year follow-up evaluation. The Journal of Arthroplasty. 1994, vol. 9, no 1, pp. 33-44, doi 10.1016/0883-5403(94)90135-х.

15. Gill T.J., Sledge J.B., Müller M.E. The Bürch-Schneider anti-protrusio cage in revision total hip arthroplasty. Indications, principles and long-term results. The Journal of Bone and Joint Surgery. British volume. 1998, vol. 80-B, no 6, pp. 946-953, doi 10.1302/0301-620x.80b6.0800946.

16. Moore M.S., McAuley J.P., Young A.M. et al. Sr. Radiographic signs of osseointegration in porouscoated acetabular components. Clinical Orthopaedics and Related Research, 2006, vol. 443, pp. 176-183, doi 10.1097/01.blo.0000201149.14078.50.

17. Gross A.E. Revision arthroplasty of the acetabulum with restoration of bone stock. Clinical Orthopaedics and Related Research, 1999, vol. 369, pp. 198-207, doi 10.1097/00003086199912000-00021. 
18. Gie G.A., Linder L., Ling R.S. et al. Impacted cancellous allografts and cement for revision total hip arthroplasty. The Journal of Bone and Joint Surgery. British volume, 1993, vol. 75-B, no 1, pp. 14-21, doi 10.1302/0301-620x.75b1.8421012.

19. Koob S., Scheidt S., Randau T.M. et al. Biological downsizing: Acetabular defectreconstruction in revision total hip arthroplasty. Der Orthopäde, 2017,vol. 46, no 2, pp. 158-167, doi: 10.1007/s00132-0163379-x.

20. Pierannunzii L., Zagra L. Bone grafts, bone graft extenders, substitutes and enhancers for acetabular reconstruction in revision total hip arthroplasty. EFORT Open Reviews, 2016 vol. 1, no 12, pp. 431-439, doi: 10.1302/2058-5241.160025.

21. Makita H., Kerboull M., Inaba Y. et al. Revision Total Hip Arthroplasty Using the Kerboull Acetabular Reinforcement Device and Structural Allograft for Severe Defects of the Acetabulum. The Journal of Arthroplasty, 2017, vol. 32, no 11, pp. 3502-3509, doi: 10.1016/j.arth.2017.06.029. 